\title{
Modulation of odour-guided behaviour in mosquitoes
}

\author{
Sharon R. Hill ${ }^{1} \cdot$ Rickard Ignell $^{1}[$ \\ Received: 25 August 2020 / Accepted: 25 November 2020 / Published online: 23 January 2021 \\ (c) The Author(s) 2021
}

\begin{abstract}
Mosquitoes are emerging as model systems with which to study innate behaviours through neuroethology and functional genomics. Decades of work on these disease vectors have provided a solid behavioural framework describing the distinct repertoire of predominantly odour-mediated behaviours of female mosquitoes, and their dependence on life stage (intrinsic factors) and environmental cues (extrinsic factors). The purpose of this review is to provide an overview of how intrinsic factors, including adult maturation, age, nutritional status, and infection, affect the attraction to plants and feeding on plant fluids, host seeking, blood feeding, supplemental feeding behaviours, pre-oviposition behaviour, and oviposition in female mosquitoes. With the technological advancements in the recent two decades, we have gained a better understanding of which volatile organic compounds are used by mosquitoes to recognise and discriminate among various fitness-enhancing resources, and characterised their neural and molecular correlates. In this review, we present the state of the art of the peripheral olfactory system as described by the neural physiology, functional genomics, and genetics underlying the demonstrated changes in the behavioural repertoire in female mosquitoes. The review is meant as a summary introduction to the current conceptual thinking in the field.
\end{abstract}

Keywords Olfaction $\cdot$ Neuroethology $\cdot$ Plant seeking $\cdot$ Host seeking $\cdot$ Oviposition

\section{Introduction}

Olfaction is pertinent to the survival and reproduction of mosquitoes, as it allows for the location and assessment of resources, including food and oviposition sites. Mosquitoes have developed a complex sensory system to detect and respond to ecologically relevant volatile organic compounds (VOCs), and the occasional volatile inorganic compounds (e.g., carbon dioxide, ammonia), in their environment. Odorants are detected by olfactory sensory neurons (OSNs) found in hair-like sensilla on the antennae, maxillary palps, and proboscis (e.g., Bohbot et al. 2013; Davis 1984a; Ghaninia et al. 2019; Kwon et al. 2006; Majeed et al. 2016; Omondi et al. 2019). The VOCs penetrate the sensilla through cuticular pores or slits, and are then transported through sensillar lymph to the OSN membranes, either by diffusion or with the aid of odorant binding proteins (OBPs), where the VOCs

Rickard Ignell

rickard.ignell@slu.se

1 Disease Vector Group, Department of Plant Protection Biology, Swedish University of Agricultural Sciences, Växtskyddsvägen 3, 23053 Alnarp, Sweden interact with odorant receptors (Ors), ionotropic receptors (Irs), or gustatory receptors (Grs) (e.g., Carey et al. 2010; Matthews et al. 2016; Omondi et al. 2015; Omondi et al. 2019; Rinker et al. 2013). The OSN signal is then transmitted to higher olfactory centres, where the processing of olfactory along with other sensory information occurs (e.g., Ghaninia et al. 2007; Haverkamp et al. 2018; Lahondère et al. 2020). At each stage in this process, the olfactory signal is available for modulation by intrinsic factors and reflected in a change in innate behaviour, as described in other insects (Gadenne et al. 2016). Innate behaviours are genetically determined and critical to the survival of the animal. While hardwired, such behaviours are available for modulation by both internal and external factors (Tinbergen 1952).

Mosquitoes display clearly staged and separated life stages, as well as well-defined host-pathogen interaction phenotypes, for which the other main model insect, Drosophila, is not suitable. As such, mosquitoes provide an excellent model system in which to study the modulation of innate behaviours, as females display a distinct repertoire of behaviours depending on life stage (intrinsic factors) and environmental cues (extrinsic factors). Building on a strong behavioural framework, we are now at a stage in which 
we, with the assistance of advanced neurophysiological and genetic techniques, are able to establish key mosquito species as model systems (Matthews and Vosshall 2020) to investigate the roots of behavioural plasticity in depth. In this review, we provide a general perspective of how adult maturation, age, and nutritional and infection status affect the attraction to plants and feeding on plant fluids, host seeking, blood feeding, supplemental feeding behaviours, pre-oviposition behaviour, and oviposition in female mosquitoes. Furthermore, we provide a current overview of these molecular and neural correlates in the context of the intrinsically regulated behaviours of mosquitoes. The authors acknowledge that this may not be a comprehensive perspective, as there are species that deviate from the perceived norm.

\section{The behavioural framework}

Mosquitoes depend on a series of innate behaviours to enhance survival and reproduction. While predominantly determined genetically, there is substantial experimental evidence demonstrating that these behaviours are affected by both intrinsic and extrinsic factors (Fig. 1). For reviews see Lyimo and Ferguson (2009) and Takken and Verhulst (2013). The authors strongly believe that an understanding of the behavioural framework, a set of clearly defined core innate behaviours that are based on laboratory and field observation data collected over the last decades on a plethora of species, is required to fully comprehend the factors contributing to the observed changes in odour-guided behaviours in mosquitoes. Further description of the modulation of these odour-guided behaviours in mosquitoes is found below.

\section{Plant attraction and feeding}

The majority of mosquito species feed from floral nectaries and other sources of plant fluids, including, e.g., extrafloral nectaries, fruits, and honeydew (Barredo and DeGennaro 2020; Foster et al. 1995; Peach and Gries 2020). The prevalence of feeding on plant fluids, however, may be affected by external factors, including, e.g., differences in habitat and the seasonal availability of plant resources, which significantly increase the observed variability in plant fluid procurement by mosquitoes (Müller and Schlein 2005; Spencer et al. 2005). For a comprehensive review of this topic, see Foster (1995). The internal state also regulates the need of female mosquitoes to feed on sugar-rich plant fluids (Fig. 1). Under natural conditions, female mosquitoes often eclose with low teneral carbohydrate and lipid energy reserves, which may be rapidly replenished through feeding on plant fluids (Briegel 1990, 2003; Foster 1995; van Handel 1965). The sugars and amino acids obtained provide energy for flight (Briegel et al. 2001; Hancock and Foster 1993; Scaraffia and Wells 2003), while the carbohydrate and lipid reserves are

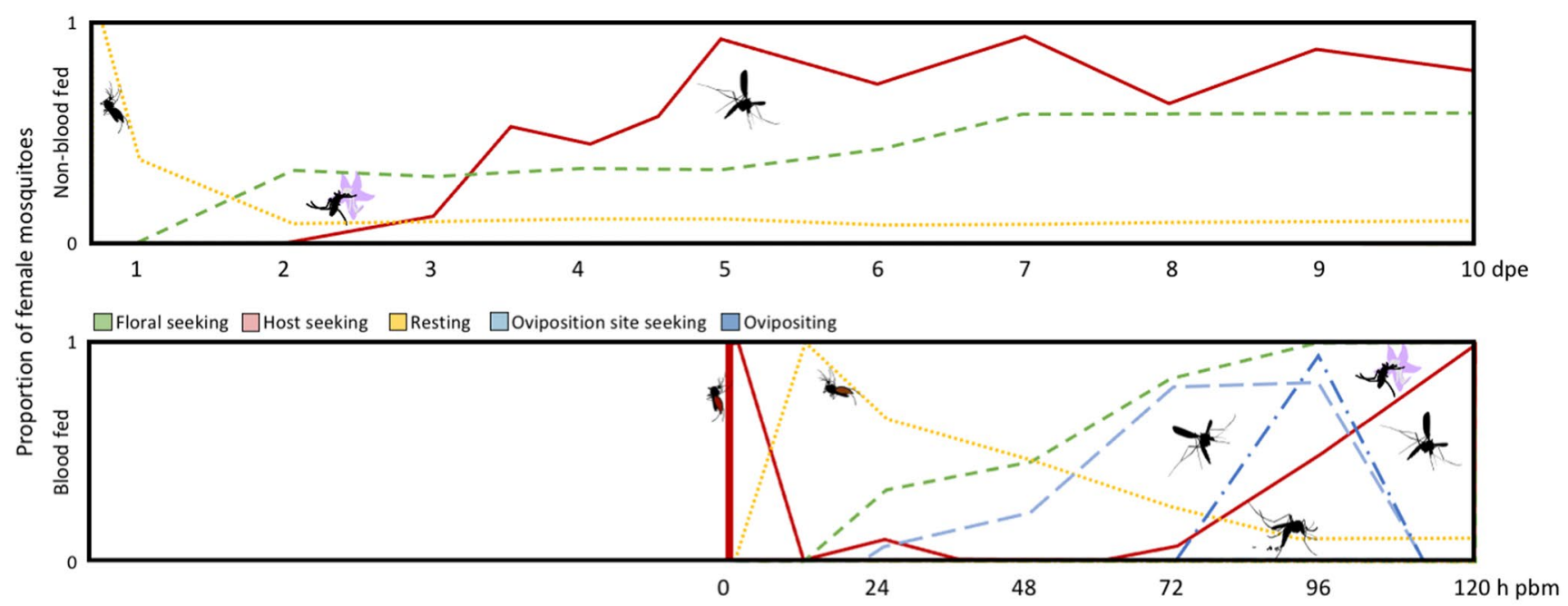

Fig. 1 Diagrammatic representation of the adult female gonotrophic cycle. After eclosion, female mosquitoes share their activities amongst floral (green, medium-dashed line) and host seeking (red, solid line), and resting (yellow, short-dashed line), as shown over the first 10-day post-eclosion (dpe; top panel). After a full blood meal (solid red vertical bar), the floral and host seeking is inhibited, the latter until after oviposition (dark blue, dash-dot line), while the former is inhibited for up to 48 -h post-blood meal (h pbm), when pre-oviposition behaviours (light blue, long-dashed line) begin (bottom panel). Most females oviposit within $96 \mathrm{~h}$ of a blood meal. The $y$-axis denotes the generalised proportion of mosquitoes participating in the associated behaviour based on, e.g., Alto et al. 2003; Bohbot et al. 2013; Christ et al. 2017; Davis 1984b; Foster 1995, references therein; Klowden 1989; Klowden 1994; Klowden and Blackmer 1987; Klowden and Lea 1984; and Vargo and Foster 1982 
used to enhance survival and reproduction (Briegel 1990; Briegel 2003; Manda et al. 2007; Moller-Jacobs et al. 2014; Nyasembe et al. 2020; Shapiro et al. 2016; Vrzal et al. 2010; Yu et al. 2016). As for other behaviours, feeding on plant fluids is endogenously regulated with many species displaying a diphasic feeding cycle (Chadee et al. 2014; Gillett et al. 1962 , and references therein). While feeding on plant fluids declines with age, there is ample evidence that females of various species frequently feed on such resources not only during the first gonotrophic cycle but also throughout adult life (Foster 1995, and references therein; Gary and Foster 2006). However, both during adult maturation and within each gonotrophic cycle, female mosquitoes undergo sequential changes in physiological condition, which affect their avidity to sugar feed (Christ et al. 2017; Foster 1995; Gary and Foster 2006). These state-dependent changes in plant feeding are reflected in the propensity of female mosquitoes to respond to attractive plant odours that are used for both location and discrimination among plants (Foster 1995, 2008; Foster and Takken, 2004; Mauer and Rowley 1999; Nyasembe et al. 2018). In contrast, the behavioural response to repellent volatile secondary plant metabolites does not appear to be state-dependently modulated (e.g., Zappia et al. 2015), although sensitivity to repellents may reduce with age of the female (Leal et al. 2017). This suggests that there is less modulation of the detection of aversive VOCs, which likely signal risk.

Following adult emergence, female mosquitoes avidly respond behaviourally to plant-derived odours and prefer these odour sources over that of vertebrate host odours (Foster 1995; Foster and Takken 2004). As females age, and their physiological state shifts, the propensity of female mosquitoes to host seek increases (Davis 1984a; Foster and Takken 2004; Omondi et al. 2019; Tallon et al. 2019), partly driven by the energy-rich meal itself, as demonstrated in various species (Briegel 2003; Foster 1995; Hancock and Foster 1997; 2000; Renshaw et al. 1995; Roitberg et al. 2010). While domestic species, including Anopheles gambiae and Aedes aegypti, are capable of deriving all required energy from human blood, and thereby of dispensing with plant feeding, individuals from these and other species often continue to be attracted and visit plants for feeding even after developing the competence to blood feed (Barredo and DeGennaro 2020; Fernandes and Briegel 2004; Foster 1995, 2008; Hancock and Foster 1993). Flower visiting, and feeding on plant fluid, however, transiently cease after females obtain a complete blood meal, reflecting the mutual inhibition of feeding responses during consumption and digestion (Christ et al. 2017; Foster 1995). Resumption of flower visiting and sugar feeding is gradual (Christ et al. 2017; Foster, 1995), and correlates with the maturation of oocytes (Vargo and Foster, 1984), in which gravid mosquitoes and females that have recently oviposited actively seek to replenish their energy reserves by increasing their flower visiting rate (Gary and Foster 2006; Magnarelli 1977; Reisen et al. 1986).

\section{Host seeking and blood feeding}

Anautogenous female mosquitoes, i.e., most mosquito species, require a vertebrate blood meal to complete egg maturation (Clements 1999). Recently emerged adult female mosquitoes are, however, incapable of taking a blood meal due to restricted function of their mouthparts and other organs (Clements 1999). Most anautogenous species develop the functional competence to blood feed between 24 and $72 \mathrm{~h}$ following adult emergence (Alto et al. 2003; Armstrong and West 1965; Clements 1999) (Fig. 1). Thereafter, blood feeding events follow an age-dependent, circadian, and diel rhythmic pattern, even in the absence of other nutrients (Alto et al. 2003; Davis 1984a; Fritz et al. 2014; Rund et al. 2016) (Fig. 1). To complete the gonotrophic cycle, female mosquitoes require a single, complete blood meal, and following this, females are generally inactive throughout the period of ovarian development and will not take another meal until after oviposition (Edman et al. 1975; Klowden and Briegel 1994; Klowden and Lea 1978; 1979a; Takken et al. 2001). This demonstrated dynamic drive to blood feed is tightly linked to the females' propensity to host seek, i.e., respond to host odour.

While the onset of the behavioural response to host odour, and other host associated stimuli, generally co-occurs with their competence to blood feed, female mosquitoes do not display full responsiveness to host odour until between 4 to 10 days after adult emergence (Bohbot et al. 2013; Davis 1984a; Foster and Takken 2004; Omondi et al. 2015, 2019; Tallon et al. 2019), after which the proportion of host-seeking mosquitoes fluctuates around a mean maximum (ca. 90\%; Alto et al. 2003; Davis 1984a). Females that have taken a full blood meal are inhibited to respond to host odour, through a two phase, immediate, and delayed process (Brown et al. 1994; Duvall et al. 2019; Klowden and Lea 1979a; 1979b). These females remain refractory to host odour, and other host associated stimuli, until after egg laying (Edman et al. 1975; Klowden and Briegel 1994; Klowden and Lea 1978; 1979a; Takken et al. 2001), at which time females actively seek new blood hosts, often after obtaining an energy-rich meal from plant fluids (Foster 1995). Such an energy-rich meal, however, appears to have no effect on the reproductive traits in anophelines, whereas it significantly reduces the overall reproductive output in Aedes species (Braks et al. 2006; Harrington et al. 2001).

Pathogens obtained through an infected blood meal may affect the propensity of female mosquitoes to host seek and blood feed in subsequent gonotrophic cycles, in a pathogenspecific pattern (Anderson et al. 1999; Grimstad et al. 1980; Koella et al. 1998, 2002; Platt et al. 1997; Tallon et al. 2020; 
Vogels et al. 2017; Wekesa et al. 1992; Yan et al. 2018). In addition, a study by Nyasembe et al. (2014) demonstrated that female An. gambiae infected with the malaria parasite Plasmodium falciparum displayed an increased attraction to floral nectar sources and sugar uptake. Moreover, Gaburro et al. (2018a, b) showed that the oviposition preference of Ae. aegypti, based on larval experience, was lost following dengue virus infection. These pathogen-induced changes in vector behaviour appear to be dependent on the progression of infection within the mosquito (Anderson et al. 1999; Koella et al. 2002; Tallon et al. 2020), and are often regarded as an active manipulation by the pathogen to increase transmission. However, other studies imply that these changes merely occur as a result of a general immunological response (Cator et al. 2013). The regulation of the olfactory behaviour of the mosquito vector by a pathogen requires further analysis and should be included in our discussions of vectorial capacity, as it increases the risk of human/ vector interactions.

\section{Supplemental feeding behaviours}

In addition to the gonotrophic cycling feeding behaviours described above, i.e., sugar and blood feeding, other conditions may arise that trigger intrinsic modulation of mosquito feeding to include supplementary meals. Emergence as an undernourished adult, restricted access to plant fluids and partial blood meals are among the factors that may force females to engage in bouts of multiple blood feeding to increase their fitness by both increasing energetic reserves and enhancing reproductive traits, e.g., increasing the number and/or size of offspring (Briegel and Hörler 1993; Farjana and Tuno 2013; Klowden and Briegel 1994; Scott and Takken 2012; Takken et al. 2002, 1998). This introduces variance in the observed innate behaviour, as shown by, e.g., Klowden and Briegel (1994), Klowden and Lea (1978, 1979b) and Takken et al. (2001). Interestingly, a meal of recently deposited cattle urine may substitute, at least in part, the role of a supplemental blood meal in host-seeking and gravid females (Dawit et al. 2018). Moreover, there are reports of mosquitoes being attracted to insect larvae and feeding on their haemolymph, which can result in the production of viable eggs (Harris et al. 1969; George et al. 2014; Martel et al. 2011). Whether there are other nitrogen-rich meals that play a similar role remains to be addressed. Following an incomplete blood meal, the host-seeking refractory period is shortened and host seeking resumed prior to oviposition, as oocyte development remains suspended until sufficient reserves are available (Farjana and Tuno 2013; Klowden and Briegel 1994; Klowden and Lea 1978; Takken et al. 2001). The resulting alterations in behaviour, following an incomplete blood meal, increases the interaction between the vector and human, thus have dire implications for disease transmission.

\section{Pre-oviposition behaviour and oviposition}

The selection of an oviposition site is critical for the reproductive success of an individual female mosquito, as well as for the population dynamics of the species. Behaviours leading up to the act of oviposition, i.e., the physical deposition of eggs, are referred to as pre-oviposition behaviours (Klowden and Blackmer 1987). When mosquitoes search for a suitable egg-laying site, females are required to make choices at increasingly finer scales during orientation, encounter, and acceptance, in which water vapour and odours play significant roles (Afify and Galizia 2015; Asmare et al. 2017; Lindh et al. 2015; Okal et al. 2013; Wondwosen et al. 2016, 2017, 2018). While pre-oviposition behaviour and its regulation have not received as much attention as plant and host seeking, the response to odours associated with these behaviours, specifically those odours emanating from oviposition sites, appears to be dependent on the physiological state of the female mosquito (Klowden 1989; Klowden and Blackmer 1987; Klowden and Dutro 1990; Manda et al. 2007).

In studies on Ae. aegypti, in which the temporal development of the pre-oviposition behaviour has been well characterised, the onset of response to oviposition-site attractants occurs $48 \mathrm{~h}$ following a complete blood meal and then reaches a maximum at 72-h post-blood feeding (Klowden and Blackmer 1987) (Fig. 1). While similar studies, to our knowledge, are lacking for other mosquito species, blood meal-induced changes in the sensitivity of OSNs (Davis and Takahashi 1980; Qiu et al. 2006; Siju et al. 2010) and in the transcript abundance of genes encoding for olfactory proteins (Rinker et al. 2013) tuned to oviposition site cues suggest a similar temporal change. With the increasing identification of ecologically relevant attractants for gravid mosquitoes (Afify and Galizia 2015; Lindh et al. 2015; Okal et al. 2013; Wondwosen et al. 2016, 2017, 2018), we are now, however, in a better position to address the time line for the behavioural changes in mosquitoes, and the factors underlying modulation of the pre-oviposition behaviour.

\section{Sensory odour space of mosquitoes}

There has been a plethora of studies on the role of odours in regulating plant-, host-, and oviposition-site seeking by female mosquitoes. For reviews see, e.g., Afify and Galizia (2015), Barredo and DeGennaro (2020), Ignell and Hill (2020), and Takken and Knols (1999). Due to recent technological advancements, and a shift in conceptual thinking concerning how insects recognise and discriminate 
resources, we have taken substantial strides forward in our understanding of the mechanisms regulating these behavioural traits from an ecological and evolutionary perspective (Ignell and Hill 2020). Despite the diversity of approaches taken to identify the behaviourally active VOCs in often complex odour sources, a number of key principles have emerged.

Mosquitoes exposed to synthetic VOCs presented in ecologically relevant blends or combinations generally display a stronger behavioural response than that to single VOCs (e.g., Ghaninia et al. 2019; Lahondére et al. 2020; Majeed et al. 2016; Nyasembe et al. 2018; Omondi et al. 2019; Takken and Knols 1999; Wondwosen et al. 2016). Moreover, recognition of resources, including plants, hosts, and oviposition sites, is contextual and dependent on quantitative and qualitative differences in blends and the olfactory codes evoked (e.g., Ghaninia et al. 2019; Lahondére et al. 2020; Majeed et al. 2016; Nyasembe et al. 2018; Omondi et al. 2019; Takken and Knols 1999; Wondwosen et al. 2016). In studies in which the release rate of individual VOCs within a blend has been controlled, mosquitoes have been shown to be attracted to a range of ecologically relevant doses (e.g., Ghaninia et al. 2019; Majeed et al. 2016; Omondi et al. 2019; Takken and Knols 1999; Wondwosen et al. 2016). However, at release rates higher than those encountered in the natural environment, mosquitoes are often either indifferent or averted by the same blends (Ghaninia et al. 2019; Majeed et al. 2016; Wondwosen et al. 2016, 2017, 2018). Changing the quality of the blend by changing the ratio of VOCs within it may also disrupt attraction (Ghaninia et al. 2019; Majeed et al. 2016), although, as with dose, there appears to be a range of tolerance. The addition of a VOC to a blend out of context often has a disruptive effect on blend attraction (Jaleta et al. 2016; Majeed et al. 2016). Seen from the perspective of odour quality perception, the behavioural response to any odour blend is a result of combinatorial coding, relying on the simultaneous detection and relative quantification of VOCs by the OSNs (Haverkamp et al. 2018; Lahondére et al. 2020; Wynne et al. 2020). Any change in the odour encountered, or in the signal relayed from the OSNs, will change the representation of a given stimulus in the higher olfactory centres and may lead to a change in the behavioural output (Haverkamp et al. 2018; Lahondère et al. 2020; Wynne et al. 2020).

With an increasing number of behaviourally active VOCs being identified, it is becoming clearer that these compounds are used parsimoniously, i.e., serve multiple functions in different behavioural contexts (Ignell and Hill 2020; Syed 2015). Chemical parsimony is reflected in the conserved function, in terms of selectivity and sensitivity to salient VOCs, of the peripheral olfactory system among widely divergent mosquito species. For reviews see, e.g., Afify and Galizia (2015), Barredo and DeGennaro (2020), Ignell and
Hill (2020), and Takken and Knols (1999). One fundamental rule underlying chemical parsimony is that the number of VOCs available for coding is constrained by the limited number of biosynthetic pathways available for production (Blum 1996). Parsimoniously used VOCs, often in combination with other VOCs, are known to elicit stereotyped behaviours, in which the blend and ratio of VOCs convey the code. From an odour coding perspective, such reliance on the combinatorial coding of parsimoniously used VOCs provides insects with the ability to adapt to a changing intrinsic and extrinsic environment. The main selection pressure is thereby proposed to be on the maintenance of the combined code in the CNS, while the peripheral detection machinery can change (e.g., substituting ligand or receptor), so long as the pattern in the CNS response is maintained within its tolerance levels.

\section{Sensory and molecular correlates of olfactory behaviour}

The modulation of olfactory behaviour in mosquitoes is underpinned by a highly plastic peripheral olfactory system (as described below), as opposed to that generally described in other insects (Gadenne et al. 2016). Over the past 30 years we have been accumulating physiological evidence emphasising that OSNs of mosquitoes alter activity in response to intrinsic factors (Bohbot et al. 2013; Davis 1984a, b; Eilerts et al. 2018; Grant and O'Connell 2007; Omondi et al. 2015; Omondi et al. 2019; Qiu et al. 2006; Rund et al. 2013b; Siju et al. 2010; Stanczyk et al. 2019; Tallon et al. 2020). With the advent of functional genomics, we are now starting to describe the molecular correlates that form the basis of the olfactory system regulation in mosquitoes. For past reviews on this topic see Criscione et al. (2015), Rinker et al. (2016) and Adelman et al. (2016). Here, we provide a current overview of what is known about the neural and molecular correlates underlying the demonstrated changes in the behavioural repertoire in female mosquitoes.

\section{Maturation and aging}

The sensitivity and specificity of the peripheral olfactory system in mosquitoes change through time (Bohbot et al. 2013; Davis 1984a; Grant and O'Connell 2007; Omondi et al. 2015, 2019; Qiu et al. 2006; Siju et al. 2010), reflecting, and likely underpinning, many of the odour-mediated behavioural shifts during maturation and aging. The period of maturation differs for various odourguided behaviours, as described above. Within minutes to hours of eclosion, once the wings have unfurled and the cuticle hardened sufficiently, mosquitoes of both sexes are competent to seek for and imbibe plant fluids, but not yet for seeking a mate or, for females, a blood meal 
(Armstrong and West 1965; Clements 1999). As of yet, we have limited understanding of the development of the OSNs and gene expression in the peripheral olfactory system within the first 24 -h post-emergence. The only study, so far, that addresses the function of OSNs within this period demonstrated that OSNs housed in grooved peg sensilla are not spontaneously active and do not respond to the host-related compound lactic acid (Davis 1984a). By 24-h post-emergence, on the other hand, these and other OSNs demonstrate spontaneous activity and sensitivity to ligands (Davis 1984a; Grant and O'Connell 2007; Omondi et al. 2015, 2019), and the majority of the genes encoding for olfactory receptors and binding proteins are expressed (Bohbot et al. 2013; Omondi et al. 2015, 2019; Tallon et al. 2019) (Fig. 2). Any gene expressed at this time point appears to be expressed throughout the adult period (Bohbot et al. 2013; Hill et al. accepted; Matthews et al. 2016; Omondi et al. 2015; Rinker et al. 2013) (Fig. 2). Only a subset of these genes is up- or downregulated genes, and none of these appear to be switched on or off (Bohbot et al. 2013; Hill et al. accepted; Matthews et al. 2016; Omondi et al. 2015; Rinker et al. 2013). Thus, by $24 \mathrm{~h}$ post-emergence, the peripheral olfactory system of the adult mosquito appears to have completed the overall development of the pattern of chemosensory gene expression.

Sexual maturity follows emergence within $18-24 \mathrm{~h}$ for males and 1-3-day post-emergence for females (Clements 1999). While sex pheromones have not yet been identified in mosquitoes, aggregation pheromones that affect the recruitment of both sexes to the mating swarm has been shown in Anopheles, and candidates proposed in Ae. aegypti (Cabrera and Jaffe 2007; Fawaz et al. 2014; Mozũraitis et al. 2020). However, there are no molecular correlates (e.g., binding proteins or receptors) for these pheromones that have been identified. The switch from the intrinsically dominant hostseeking behaviour to mating appears to be a result of sexually mature females repressing the expression of a subset of genes involved in host seeking following their exposure to sexually mature males, which is reversed following insemination (Alonso et al. 2019; Jones 1981; Jones and Gubbins 1978).

Female mosquitoes begin to develop the ability to seek for a blood host from as young as $1 \mathrm{~d}$ post-emergence, and continue to mature for up to 10-day post-emergence (Alto et al. 2003; Davis 1984a; Omondi et al. 2015, 2019; Tallon et al. 2019). While both OSN sensitivity and olfactory-related gene expression generally increase as females mature to hostseeking age (Bohbot et al. 2013; Davis 1984a; Grant and
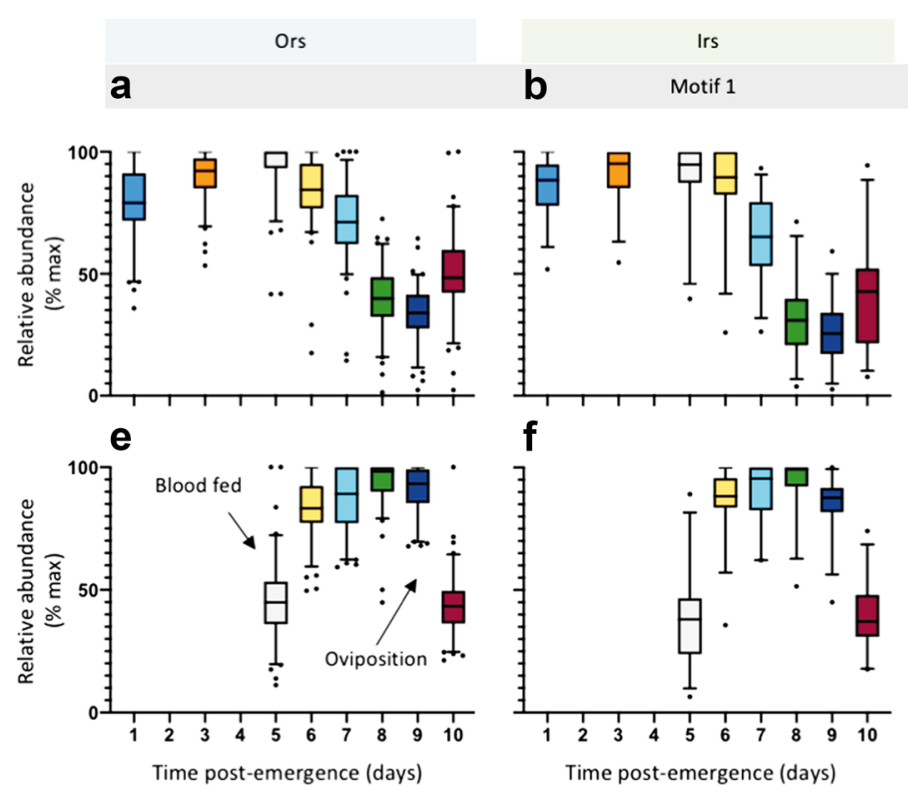

C

OBPs
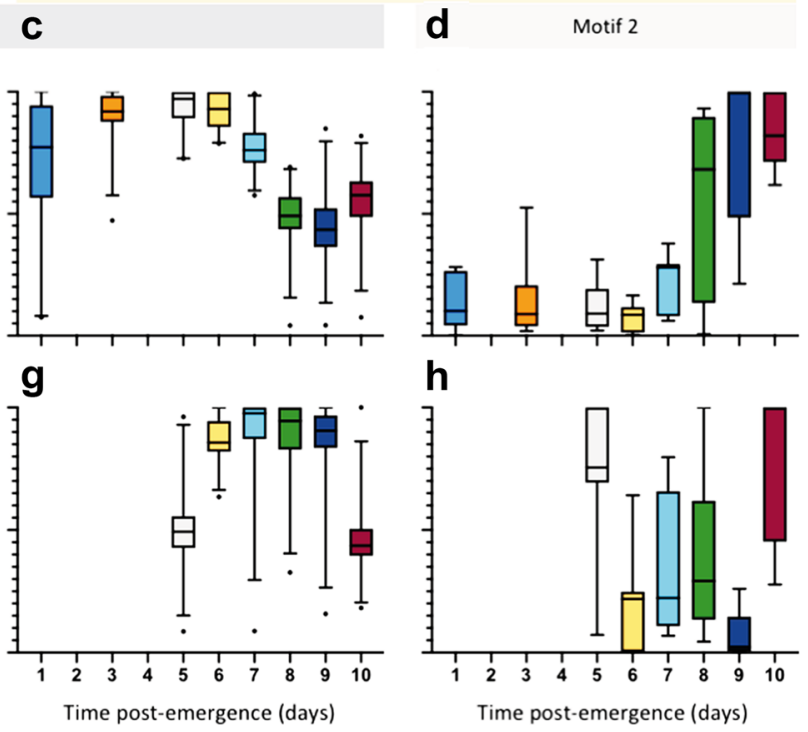

Fig. 2 Patterns of antennal transcript abundance for select chemosensory gene families depicted during the first gonotrophic cycle of a female mosquito. The relative transcript abundance, as determined as the percent of the maximal average abundance over time (days) for each gene of the odorant receptor (Or, a, e), ionotropic receptor ( $\mathrm{Ir}$, $\mathbf{b}, \mathbf{f}$ ), and odorant binding protein ( $\mathrm{OBP}, \mathbf{c}, \mathbf{d}, \mathbf{g}, \mathbf{h}$ ) families, in the antennae of female Aedes aegypti are shown in box plots, with the whiskers denoting 5-95 percentile. Antennal transcript profiles were made from females with ad libitum access to sugar, and either no

access to blood (a-d) or a complete blood meal at 5-day post-emergence $(\mathbf{e}-\mathbf{h})$. There are two evident motifs in transcript abundance, motif 1 , as shown by the receptor families $(\mathbf{a}, \mathbf{b}, \mathbf{e}, \mathbf{f})$ and the majority of the expressed OBPs $(\mathbf{c}, \mathbf{g})$, and motif 2, as observed in the remaining $20 \%$ (d, h). Data presented here originate from Tallon et al. (2019) and Hill et al. (accepted). Note that data was not collected on days 2 and 4 for non-blood fed females, and as females were fed on day 5 , no data is shown in days $1-4$ for blood fed females 
O'Connell 2007; Hill et al. accepted; Omondi et al. 2015; Omondi et al. 2019; Tallon et al. 2019) (Fig. 2), there are a few notable exceptions, which has been postulated to gate the switch from pre-host seeking to host seeking (Omondi et al. 2019; Tallon et al. 2019). For example, the host responsiveness of 1-day and 4-day post-emergence female Anopheles coluzzii has been demonstrated to correlate with the reduction in OSN sensitivity to the host cue sulcatone and the decreased abundance of its cognate odorant receptor, Or39, with age (Omondi et al. 2019). Moreover, an odorant receptor, $\operatorname{Or} 117$, and two odorant binding proteins, Obp22 and Obp64, with a similar expression pattern to An. coluzzii Or39 have been described for Ae. aegypti (Tallon et al. 2019). While the ligand specificities of these proteins are not yet known, these may serve a similar function as proposed for An. coluzzii. With the availability of gene editing tools, we are now in a position to assess whether the observed expression pattern of chemosensory genes and changes in sensitivity of OSNs during maturation regulate the onset of agedependent behaviours, e.g., host seeking and mating.

While the onset of host seeking in many anautogenous mosquito species appears to be completed within a week of eclosion (Clements 1999; Davis 1984a), the propensity to engage in the behaviour appears to oscillate as the female ages (Alto et al. 2003), as does the abundance of a subset of olfactory-related genes (Hill et al. accepted). Such oscillations may represent opportunities within the host-seeking phase of the gonotrophic cycle for the female mosquitoes to replenish dwindling energy reserves by engaging in plant-seeking activities (Alto et al. 2003; Hill et al. accepted). In general, as female mosquitoes age, the incidence of plant feeding decreases and host seeking increases (Foster 1995; Gary and Foster 2006). Few studies have investigated the sensitivity of OSNs or the transcript abundance in the peripheral olfactory system of females in older host-seeking females, and these data are largely collected as controls for assessing state-dependent change (Davis 1984a; Bohbot et al. 2013; Hill et al. accepted; Tallon et al. 2020), as there is low probability of finding older ( $>10$-day post-emergence) nulliparous females in the wild. Collectively, available data suggests that host seeking is regulated and modulated as the female ages, and this is, at least in part, due to changes in expression of the chemosensory-related genes in the peripheral olfactory system (Fig. 2). Future functional studies are, however, required to elucidate the molecular underpinnings of the periodic agedependent behaviours.

\section{Circadian and daily rhythm effects}

The cycling of odour-mediated behaviour throughout the day is reflected in the neural and molecular correlates of the peripheral nervous system in mosquitoes (Das De et al. 2018; Eilerts et al. 2018; Rund et al. 2011, 2013a, b). The sensitivity of the peripheral olfactory system to various ecologically important VOCs changes in a daily rhythm in both An. gambiae (Rund et al. 2013b) and Ae. aegypti (Eilerts et al. 2018). Of the responses to VOCs that cycle, the peripheral olfactory system is most sensitive to VOCs that induce attraction when assessed as single compounds during the peak activity periods in both species (Rund et al. 2013b; Eilerts et al. 2018). This includes the sensitivity of OSNs tuned to the ubiquitous host volatile inorganic compound, carbon dioxide (Siju, Hill and Ignell, unpublished data). The VOCs that are avoided when tested as single compounds, on the other hand, are detected with the highest sensitivity preand post-activity peak (Eilerts et al. 2018), suggesting a role for these VOCs in regulating the daily onset and cessation of odour-mediated activities. It is important to note that VOCs may display corresponding cycling of emissions, e.g., floral scent (for review see, e.g., Fenske and Imaizumi 2016). Further investigation of the cycling of OSN sensitivity to ecologically relevant VOCs, and blends thereof, at naturally fluctuating release rates, is required to fully comprehend the correlation with demonstrated rhythmic behaviours.

Cycling of chemosensory gene expression, protein translation, and degradation plays a role in generating the daily cycling in OSN sensitivity and odour-mediated behaviour of female mosquitoes (Rund et al. 2011, 2013a, b; Das De et al. 2018). The expression of at least $15 O B P$ s, 60 putative odorant degradation enzymes (ODEs), 4 class B scavenger receptors ( $S C R B \mathrm{~s}$ ), including $S N M P 1$, and the $O r$ co-receptor $($ Orco $)$, cycle in a daily rhythm in the heads of hostseeking An. gambiae females (Rund et al. 2011, 2013a). Proteomic analysis of these females' antennae revealed 12 OBPs rhythmically cycling in protein abundance, offset from the transcript abundance cycle by $9-10 \mathrm{~h}$, with 11 OBPs peaking in abundance during the scotophase period of maximal OSN sensitivity and odour-mediated activity, clearly indicating a role of OBPs in the regulation of odour-mediated daily rhythms (Rund et al. 2013b). While the transcript abundance of Orco cycles with a peak similar to that of the majority of the cycling OBPs in the heads (Rund et al. 2011, 2013a), Orco did not cycle significantly in the proteomic study of the antennae (Rund et al. 2013b). The expression level of membrane proteins in the antennae is low, and as such, Orco was the only Or to be identified (Rund et al. 2013b). Thus, further investigation is required to determine whether Ors play a role in the daily rhythm of the peripheral olfactory system and odour-driven behaviours.

Daily rhythms in olfactory-related gene expression demonstrate three types of regulation by the entrainment to light and/or by the internal molecular clock of an organism (Rund et al. 2013a). Type I genes (e.g., OBPO) require both the internal molecular clock and entrainment to light to maintain the daily rhythm, and when deprived of light will "run down" over time (Rund et al. 2013a). Type II genes (e.g., $O B P 2, S N M P 1$ ) are primarily regulated by the 
internal molecular clock; however, the level of expression is enhanced in the presence of light (Rund et al. 2013a). Finally, type III genes (e.g., $O B P 51$ ) are regulated entirely by entrainment to the light, and when placed in constant darkness will immediately lose rhythmicity (Rund et al. 2013a). Several cis-response elements demonstrate a degree of correlation with each of the three types of rhythmic regulation underlying the function of the peripheral olfactory system of the mosquito, suggesting a complex interaction among the circadian and photic signalling pathways regulating odour-driven behaviours in mosquitoes (Rund et al. 2013a).

\section{The effect of a blood meal}

As described above, the advent of a blood meal alters the behaviour of a female mosquito, and appears to be modulated, at least in part, by the peripheral olfactory system. A subset of antennal and maxillary palp OSNs change in sensitivity post-blood meal, both increasing and decreasing in response to a subset of cognate ligands (Davis 1984b; Hill et al. 2019; Qiu et al. 2006; Siju et al. 2010). Investigations into the molecular basis of this modulation of OSN sensitivity have assessed the abundance of antennal and maxillary palp gene transcripts, pre- and post-blood meal, in the females of several species, and have also identified subsets of blood meal regulated chemosensory genes (Hill et al. 2019; Hill et al. accepted; Matthews et al. 2016; Rinker et al. 2013; Taparia et al. 2017) (Fig. 2).

Time post-blood meal is another factor, in addition to blood meal, that modulates OSN sensitivity (Davis 1984b; Davis and Takahashi 1980; Qiu et al. 2006; Siju et al. 2010), chemosensory transcript abundance (Hill et al. accepted; Matthews et al. 2016; Rinker et al. 2013) (Fig. 2), and odour-driven behaviours. Immediately following a blood meal, few of the olfactory-related transcripts change significantly in abundance (Hill et al. accepted; Matthews et al. 2016; Rinker et al. 2013), suggesting that the cessation of host seeking after a blood meal partly relies on a limited number of olfactoryrelated genes in the peripheral olfactory system. Between 1- to 3-day post-blood meal, a small subset of these genes appears to be regulated (Hill et al. 2019; Hill et al. accepted; Matthews et al. 2016; Rinker et al. 2013), which may be correlated with the gradual restoration of sugar feeding (Christ et al. 2017). However, 96-h post-blood feeding, the majority of these genes in the antennae of Ae. aegypti follow one of two profiles, either a significantly higher (motif 1 ) or a significantly lower (motif 2) transcript abundance (Matthews et al. 2016; Hill et al. accepted) (Fig. 2), which correlates with the time when females are engaged in pre-oviposition behaviours. Postoviposition, the abundance of chemosensory-related genes appears to return to host-seeking levels (Hill et al. accepted) (Fig. 2). Similar to the regulation described for the diel and circadian cycling olfactory-related genes (see above), several cis-regulatory elements correlate to each of the two age- and state-dependent motifs (Hill et al. accepted). This suggests that signalling pathways associated with the monitoring of age, feeding, and reproductive state may modulate odour-driven behaviours in mosquitoes through, as yet undescribed, transcriptional regulatory pathways.

To date, there has only been a single attempt to correlate the Or ligand sensitivity (Carey et al. 2010; Wang et al. 2010) with transcript abundance pre- and post-blood meal in $A n$. gambiae females (Rinker et al. 2013), to produce a profile of the predicted change in antennal receptivity to these ligands over the first $48 \mathrm{~h}$ after blood feeding (Rinker et al. 2013). In looking at the antennal response as a whole, we are able to observe an immediate increase in sensitivity to one subset of VOCs post-blood meal, followed by an increase in sensitivity to another subset of compounds $24 \mathrm{~h}$ later (Rinker et al. 2013). The ongoing functional characterisation of Ors in various mosquito species is likely to shed light on the relevance of these changes in transcript abundance for behavioural modulation.

\section{Infection}

Pathogen-infected mosquitoes often exhibit altered odourmediated behaviours (Koella et al. 2002; Nyasembe et al. 2014; Gaburro et al. 2018a, b; Tallon et al. 2020), corresponding with post-infection changes in the sensitivity of the peripheral olfactory system to behaviourally relevant odorants (Stanczyk et al. 2019; Tallon et al. 2020) and neural activity patterns in the brain (Salazar et al. 2007). Moreover, the abundance of many transcripts related to neural signalling change in response to pathogen infection in the antennae, heads, and bodies of mosquitoes (Emami et al. 2017; Gaburro et al. 2018a, b; Salazar et al. 2007; Tallon et al. 2020), suggesting that neural signalling pathways may be one target of pathogen-induced modulation. In addition, a limited number of olfactory-related transcripts alter abundance in the antennae of host-seeking females capable of transmitting the pathogen (Tallon et al. 2020), indicating that the regulation of these chemosensory transcripts is another target of pathogen manipulation. Both of these changes in gene expression in response to pathogen infection may play a role in increasing the likelihood of disease transmission through the manipulation of coding in the olfactory system.

\section{Conclusion}

From the state of the art in mosquito neuroethology and functional genomics, these insects are emerging as models for the investigation of the mechanisms underpinning behavioural plasticity. With the ongoing functional characterisation of the sensory and molecular correlates of the peripheral olfactory system, and how these affect the behaviour, 
in important disease vector mosquitoes, An. gambiae sensu lato, Ae. aegypti, and Culex quinquefasciatus, we are creating a solid foundation for further understanding of the mechanisms regulating the diverse repertoire of behaviours that dictate vectorial capacity. With such models, we are better positioned to identify novel targets for vector control.

Acknowledgments The authors would like to thank current and previous members of our group, our colleagues, and the Swedish research councils, Vetenskapsrådet and Formas, for their generous long-term financial support.

Funding Open Access funding provided by Swedish University of Agricultural Sciences.

Open Access This article is licensed under a Creative Commons Attribution 4.0 International License, which permits use, sharing, adaptation, distribution and reproduction in any medium or format, as long as you give appropriate credit to the original author(s) and the source, provide a link to the Creative Commons licence, and indicate if changes were made. The images or other third party material in this article are included in the article's Creative Commons licence, unless indicated otherwise in a credit line to the material. If material is not included in the article's Creative Commons licence and your intended use is not permitted by statutory regulation or exceeds the permitted use, you will need to obtain permission directly from the copyright holder. To view a copy of this licence, visit http://creativecommons.org/licenses/by/4.0/.

\section{References}

Adelman ZN, Basu S, Myles KM (2016) Gene insertion and deletion in mosquitoes. In: Adelman ZN (ed) Genetic control of malaria and dengue Academic Press London 139168

Afify A, Galizia CG (2015) Chemosensory cues for mosquito oviposition site selection. J Med Entomol 52:120-130

Alonso DP, Campos M, Troca H, Kunii R, Tripet F, Ribolla PE et al (2019) Gene expression profile of Aedes aegypti females in courtship and mating. Sci Rep 9:15492

Alto BW, Lounibos LP, Juliano SA et al (2003) Age-dependent bloodfeeding of Aedes aegypti and Aedes albopictus on artificial and living hosts. J Am Mosq Control Assoc 19:347-352

Anderson RA, Koella JC, Hurd H et al (1999) The effect of Plasmodium yoelii nigeriensis infection on the feeding persistence of Anopheles stephensi Liston throughout the sporogonic cycle. Proc R Soc B Biol Sci 266:1729-1733

Armstrong JA, West AS (1965) Blood feeding activity by newly emerged female mosquitoes. Mosq News 25:263-268

Asmare Y, Hill SR, Hopkins RJ, Tekie H, Ignell R et al (2017) The role of grass volatiles on oviposition site selection by Anopheles arabiensis and Anopheles coluzzii. Malar J 16:65

Barredo E, DeGennaro M (2020) Not just from blood: Mosquito nutrient acquisition from nectar sources. Trends Parasitol 36:473-484

Blum MS (1996) Semiochemical parsimony in the Arthropoda. Ann Rev Entomol 41:353-374

Bohbot JD, Durand NF, Vinyard BT, Dickens JC et al (2013) Functional development of the octenol response in Aedes aegypti. Front Physiol 4:39

Braks MA, Juliano SA, Lounibos LP et al (2006) Superior reproductive success on human blood without sugar is not limited to highly anthropophilic mosquito species. Med Vet Entomol 20:53-59

Briegel H (1990) Fecundity, metabolism, and body size in Anopheles (Diptera: Culicidae), vectors of malaria. J Med Entomol 27(5):839-850
Briegel H (2003) Physiological bases of mosquito ecology. J Vector Ecol 28:1-11

Briegel H, Knusel I, Timmermann SE et al (2001) Aedes aegypti: Size, reserves, survival, and flight potential. J Vector Ecol 26:21-31

Briegel H, Hörler E (1993) Multiple blood meals as a reproductive strategy in Anopheles (Diptera: Culicidae). J Med Entomol 30(6):975-985

Brown MR, Klowden MJ, Crim JW, Young L, Shrouder LA, Lea AO et al (1994) Endogenous regulation of mosquito host-seeking behavior by a neuropeptide. J Insect Physiol 40:399-406

Cabrera M, Jaffe K (2007) An aggregation pheromone modulates lekking behavior in the vector mosquito Aedes aegypti (Diptera: Culicidae). J Am Mosquito Contr 23:1-10

Carey AF, Wang G, Su CY, Zwiebel LJ, Carlson JR et al (2010) Odorant reception in the malaria mosquito Anopheles gambiae. Nature 464:66-71

Cator LJ, George J, Blanford S, Murdock CC, Baker TC, Read AF, Thomas MB et al (2013) "Manipulation" without the parasite: Altered feeding behaviour of mosquitoes is not dependent on infection with malaria parasites. Proc R Soc B Biol Sci 280:20130711

Chadee DD, Sutherland JM, Gilles JR et al (2014) Diel sugar feeding and reproductive behaviours of Aedes aegypti mosquitoes in Trinidad: with implications for mass release of sterile mosquitoes. Act Trop 132:S86-90

Christ P, Reifenrath A, Kahnt J, Hauser F, Hill SR, Schachtner J, Ignell $\mathrm{R}$ et al (2017) Feeding-induced changes in allatostatin$\mathrm{A}$ and short neuropeptide $\mathrm{F}$ in the antennal lobes affect odormediated host seeking in the yellow fever mosquito. Aedes aegypti PloS One 12:e188243

Clements AN (1999) The biology of mosquitoes. Volume 2: Sensory reception and behaviour. CABI publishing; Wallingford.

Criscione F, O'Brochta DA, Reid W et al (2015) Genetic technologies for disease vectors. Curr Opin Insect Sci 10:90-97

De Das T, Thomas T, Verma S, Singla D, Chauhan C, Srivastava V, Sharma P, Kumari S, Tevatiya S, Rani J, Hasija Y et al (2018) A synergistic transcriptional regulation of olfactory genes drives blood-feeding associated complex behavioral responses in the mosquito Anopheles culicifacies. Front Physiol 9:577

Davis EE (1984a) Development of lactic acid-receptor sensitivity and host-seeking behaviour in newly emerged female Aedes aegypti mosquitoes. J Insect Physiol 30:211-215

Davis EE (1984b) Regulation of sensitivity in the peripheral chemoreceptor systems for host-seeking behaviour by a haemolymphborne factor in Aedes aegypti. J Insect Physiol 30:179-183

Davis EE, Takahashi FT (1980) Humoral alteration of chemoreceptor sensitivity in the mosquito. Olfaction and taste 4:139-142

Dawit MB (2018) The role of cattle urine as a host habitat cue and nutrient resource for malaria mosquitoes. $\mathrm{PhD}$ thesis. Addis Ababa University.

Duvall LB, Ramos-Espiritu L, Barsoum KE, Glickman JF, Vosshall LB et al (2019) Small-molecule agonists of Ae. aegypti neuropeptide Y receptor block mosquito biting. Cell 76:687-701

Edman JD, Cody E, Lynn H et al (1975) Blood-feeding activity of partially engorged Culex nigripalpus (Diptera: Culicidae). Entomol Exp Appl 18:261-268

Eilerts DF, VanderGiessen M, Bose EA, Broxton K, Vinauger C et al (2018) Odor-specific daily rhythms in the olfactory sensitivity and behavior of Aedes aegypti mosquitoes. Insects 9:147

Emami SN, Lindberg BG, Hua S, Hill SR, Mozuraitis R, Lehmann P, Birgersson G, Borg-Karlson AK, Ignell R, Faye I et al (2017) A key malaria metabolite modulates vector blood seeking, feeding, and susceptibility to infection. Science 355:1076-1080

Farjana T, Tuno N (2013) Multiple blood feeding and host-seeking behavior in Aedes aegypti and Aedes albopictus (Diptera: Culicidae). J Med Entomol 50:838-846 
Fawaz EY, Allan SA, Bernier UR, Obenauer PJ, Diclaro JW et al (2014) Swarming mechanisms in the yellow fever mosquito: Aggregation pheromones are involved in the mating behavior of Aedes aegypti. J Vector Ecol 39:347-354

Fenske MP, Imaizumi T (2016) Circadian rhythms in floral scent emission. Front Plant Sci 7:462

Fernandes L, Briegel H (2004) Reproductive physiology of Anopheles gambiae and Anopheles atroparvus. J Vector Ecol 30:11-26

Foster WA (1995) Mosquito sugar feeding and reproductive energetics. Ann Rev Entomol 40:443-474

Foster WA (2008) Phytochemicals as population sampling lures. J Am Mosq Contr 24:138-146

Foster WA, Takken W (2004) Nectar-related vs. human-related volatiles: behavioural response and choice by female and male Anopheles gambiae (Diptera: Culicidae) between emergence and first feeding. Bull Entomol Res 94:145-157

Fritz ML, Walker ED, Yunker AJ, Dworkin I et al (2014) Daily blood feeding rhythms of laboratory-reared North American Culex pipiens. J Circ Rhythms 12:1

Gaburro J, Bhatti A, Harper J, Jeanne I, Dearnley M, Green D, Nahavandi S, Paradkar PN, Duchemin JB et al (2018) Neurotropism and behavioral changes associated with Zika infection in the vector Aedes aegypti. Emerg Microbes Infect 7:68

Gaburro J, Paradkar PN, Klein M, Bhatti A, Nahavandi S, Duchemin $\mathrm{J}$ et al (2018) Dengue virus infection changes Aedes aegypti oviposition olfactory preferences. Sci Rep 8:13179

Gadenne C, Barrozo RB, Anton S et al (2016) Plasticity in insect olfaction: to smell or not to smell? Ann Rev Entomol 61:317-333

Gary RE Jr, Foster WA (2006) Diel timing and frequency of sugar feeding in the mosquito Anopheles gambiae, depending on sex, gonotrophic state and resource availability. Med Vet Entomol 20:308-316

George J, Blanford S, Thomas MB, Baker TC et al (2014) Malaria mosquitoes host-locate and feed upon caterpillars. PLoS ONE 9:e108894

Ghaninia M, Ignell R, Hansson BS et al (2007) Functional classification and central nervous projections of olfactory receptor neurons housed in antennal trichoid sensilla of female yellow fever mosquitoes, Aedes aegypti. Eur J Neurosci 26:1611-1623

Ghaninia M, Majeed S, Dekker T, Hill SR, Ignell R et al (2019) Hold your breath - differential behavioral and sensory acuity of mosquitoes to acetone and carbon dioxide. PLoS ONE 14:e0226815

Gillett JD, Haddow AJ, Corbet PS et al (1962) The sugar-feedingcycle in a cage-population of mosquitoes. Entomol Exp Appl 5:223-232

Grant AJ, O'Connell RJ (2007) Age-related changes in female mosquito carbon dioxide detection. J Med Entomol 44:617-623

Grimstad PR, Ross QE, Craig GB Jr et al (1980) Aedes triseriatus (Diptera: Culicidae) and La Crosse virus II. Modification of mosquito feeding behavior by virus infection. J Med Entomol 17:1-7

Hancock RG, Foster WA (1993) Effects of preblood-meal sugar on sugar seeking and upwind flight by gravid and parous Aedes aegypti (Diptera: Culicidae). J Med Entomol 30:353-359

Hancock RG, Foster WA (1997) Larval and adult nutrition effects on blood/nectar choice of Culex nigripalpus mosquitoes. Med Vet Entomol 11:112-122

Hancock RG, Foster WA (2000) Exogenous juvenile hormone and methoprene, but not male accessory gland substances or ovariectomy, affect the blood/nectar choice of female Culex nigripalpus mosquitoes. Med Vet Entomol 14:376-382

Harrington LC, Edman JD, Scott TW et al (2001) Why do female Aedes aegypti (Diptera: Culicidae) feed preferentially and frequently on human blood? J Med Entomol 38:411-422

Harris P, Riordan DF, Cooke D et al (1969) Mosquitoes feeding on insect larvae. Science 164:184-185
Haverkamp A, Hansson BS, Knaden M et al (2018) Combinatorial codes and labeled lines: how insects use olfactory cues to find and judge food, mates, and oviposition sites in complex environments. Front Physiol 9:49

Hill SR, Ghaninia M, Ignell R et al (2019) Blood meal induced regulation of gene expression in the maxillary palps, a chemosensory organ of the mosquito Aedes aegypti. Front Ecol Evol 7:336

Hill SR, Taparia T, Ignell R (In press) Regulation of the antennal transcriptome of the dengue vector, Aedes aegypti, during the first gonotrophic cycle. BMC Genomics

Ignell R, Hill SR (2020) Malaria mosquito chemical ecology. Curr Opin Insect Sci 40:6-10

Jaleta KT, Hill SR, Birgersson G, Tekie H, Ignell R et al (2016) Chicken volatiles repel host-seeking malaria mosquitoes. Malar J 15:354

Jones MD (1981) The programming of circadian flight-activity in relation to mating and the gonotrophic cycle in the mosquito, Aedes aegypti. Physiol Entomol 6:307-313

Jones MD, Gubbins SJ (1978) Changes in the circadian flight activity of the mosquito Anopheles gambiae in relation to insemination, feeding and oviposition. Physiol Entomol 3:213-220

Klowden MJ (1989) Influence of the ovaries and fat body on the initiation and termination of pre-oviposition behavior in the mosquito, Aedes aegypti. J Insect Physiol 35:567-570

Klowden MJ (1994) Endogenous regulation of the attraction of Aedes aegypti mosquitoes. J Am Mosq Contr 10:326-332

Klowden MJ, Blackmer JL (1987) Humoral control of pre-oviposition behaviour in the mosquito, Aedes aegypti. J Insect Physiol 33:689-692

Klowden MJ, Briegel H (1994) Mosquito gonotrophic cycle and multiple feeding potential: contrasts between Anopheles and Aedes (Diptera: Culicidae). J Med Entomol 31:618-622

Klowden MJ, Dutro SM (1990) Effects of carbohydrate ingestion on the pre-oviposition behavior of the mosquito Aedes aegypti (L.). Bull Soc Vector Ecol 15:59-62

Klowden MJ, Lea AO (1978) Blood meal size as a factor affecting continued host-seeking by Aedes aegypti (L.). Am J Trop Med Hyg 27:827-831

Klowden MJ, Lea AO (1979a) Humoral inhibition of host-seeking in Aedes aegypti during oöcyte maturation. J Insect Physiol 25:231-235

Klowden MJ, Lea AO (1979b) Abdominal distention terminates subsequent host-seeking behaviour of Aedes aegypti following a blood meal. J Insect Physiol 25:583-585

Klowden MJ, Lea AO (1984) Blood feeding affects age-related changes in the host-seeking behavior of Aedes aegypti (Diptera: Culicidae) during oocyte maturation. J Med Entomol 21:274-277

Koella JC, Rieu L, Paul REL et al (2002) Stage-specific manipulation of a mosquito's host-seeking behavior by the malaria parasite Plasmodium gallinaceum. Behav Ecol 13:816-820

Koella JC, Sorensen FL, Anderson RA et al (1998) The malaria parasite, Plasmodium falciparum, increases the frequency of multiple feeding of its mosquito vector, Anopheles gambiae. Proc R Soc B Biol Sci 265:763-768

Kwon HW, Lu T, Rützler M, Zwiebel LJ et al (2006) Olfactory responses in a gustatory organ of the malaria vector mosquito Anopheles gambiae. Proc Natl Acad Sci 103:13526-13531

Lahondère C, Vinauger C, Okubo RP, Wolff GH, Chan JK, Akbari OS, Riffell JA et al (2020) The olfactory basis of orchid pollination by mosquitoes. Proc Natl Acad Sci 117:708-716

Leal WS, Barbosa RM, Zeng F, Faierstein GB, Tan K, Paiva MH, Guedes DR, Crespo MM, Ayres CF et al (2017) Does Zika virus infection affect mosquito response to repellents? Sci Rep 7:42826

Lindh JM, Okal MN, Herrera-Varela M, Borg-Karlson AK, Torto B, Lindsay SW, Fillinger U et al (2015) Discovery of an oviposition 
attractant for gravid malaria vectors of the Anopheles gambiae species complex. Malar J 14:119

Lyimo IN, Ferguson HM (2009) Ecological and evolutionary determinants of host species choice in mosquito vectors. Trends Parasitol 25:189-196

Magnarelli LA (1977) Nectar feeding by Aedes sollicitans and its relation to gonotrophic activity. Environ Entomol 6:237-242

Majeed S, Hill SR, Birgersson G, Ignell R et al (2016) Detection and perception of generic host volatiles by mosquitoes modulate host preference: context dependence of $(R)$-1-octen-3-ol. R Soc Open Sci 3:160467

Manda H, Gouagna LC, Foster WA, Jackson RR, Beier JC, Githure JI, Hassanali A et al (2007) Effect of discriminative plant-sugar feeding on the survival and fecundity of Anopheles gambiae. Malar J 6:113

Martel V, Schlyter F, Ignell R, Hansson BS, Anderson P et al (2011) Mosquito feeding affects larval behaviour and development in a moth. PLoS ONE 6:e25658

Matthews BJ, Vosshall LB (2020) How to turn an organism into a model organism in 10 'easy'steps. J Exp Biol 223:jeb218198

Matthews BJ, McBride CS, DeGennaro M, Despo O, Vosshall LB et al (2016) The neurotranscriptome of the Aedes aegypti mosquito. BMC Genomics 17:32

Mauer DJ, Rowley WA (1999) Attraction of Culex pipiens pipiens (Diptera: Culicidae) to flower volatiles. J Med Entomol 36:503-507

Moller-Jacobs LL, Murdock CC, Thomas MB et al (2014) Capacity of mosquitoes to transmit malaria depends on larval environment. Parasit Vector 7:593

Mozūraitis R, Hajkazemian M, Zawada JW, Szymczak J, Pålsson K, Sekar V, Biryukova I, Friedländer MR, Koekemoer LL, Baird JK, Borg-Karlson AK et al (2020) Male swarming aggregation pheromones increase female attraction and mating success among multiple African malaria vector mosquito species. Nat Ecol Evol 3:1-7

Müller G, Schlein Y (2005) Plant tissues: The frugal diet of mosquitoes in adverse conditions. Med Vet Entomol 19:413-422

Nyasembe VO, Tchouassi DP, Pirk CW, Sole CL, Torto B et al (2018) Host plant forensics and olfactory-based detection in Afro-tropical mosquito disease vectors. PLoS Neglect Trop D 12:e0006185

Nyasembe VO, Tchouassi DP, Muturi MN, Pirk CW, Sole CL, Torto B (2020) Plant nutrient quality promotes survival and reproductive fitness of the dengue vector Aedes aegypti. https://doi. org/10.21203/rs.3.rs-34739/v1

Nyasembe VO, Teal PE, Sawa P, Tumlinson JH, Borgemeister C, Torto B et al (2014) Plasmodium falciparum infection increases Anopheles gambiae attraction to nectar sources and sugar uptake. Curr Biol 24:217-221

Okal MN, Francis B, Herrera-Varela M, Fillinger U, Lindsay SW et al (2013) Water vapour is a pre-oviposition attractant for the malaria vector Anopheles gambiae sensu stricto. Malar J 12:365

Omondi AB, Ghaninia M, Dawit M, Svensson T, Ignell R et al (2019) Age-dependent regulation of host seeking in Anopheles coluzzii. Sci Rep 9:9699

Omondi BA, Majeed S, Ignell R et al (2015) Functional development of carbon dioxide detection in the maxillary palp of Anopheles gambiae. J Exp Biol 218:2482-2488

Peach DA, Gries G (2020) Mosquito phytophagy-sources exploited, ecological function, and evolutionary transition to haematophagy. Entomol Exp Appl 168:120-136

Platt KB, Linthicum KJ, Myint KSA, Innis BL, Lerdthusnee K, Vaughn DW et al (1997) Impact of dengue virus infection on feeding behavior of Aedes aegypti. Am J Trop Med Hyg 57:119-125

Qiu YT, Van Loon JJ, Takken W, Meijerink J, Smid HM et al (2006) Olfactory coding in antennal neurons of the malaria mosquito, Anopheles gambiae. Chem Senses 31:845-863
Reisen WK, Meyer RP, Milby MM et al (1986) Patterns of fructose feeding by Culex tarsalis (Diptera: Culicidae). J Med Entomol 23:366-373

Renshaw M, Silver JB, Service MW (1995) Differential lipid reserves influence host-seeking behaviour in the mosquitoes Aedes cantans and Aedes punctor. Med Vet Entomol 9:381-387

Rinker DC, Pitts RJ, Zhou X, Suh E, Rokas A, Zwiebel LJ et al (2013) Blood meal-induced changes to antennal transcriptome profiles reveal shifts in odor sensitivities in Anopheles gambiae. Proc Natl Acad Sci 110:8260-8265

Rinker DC, Pitts RJ, Zwiebel LJ et al (2016) Disease vectors in the era of next generation sequencing. Genome Biol 17:95

Roitberg BD, Keiser S, Hoffmeister T et al (2010) State-dependent attacks in a mosquito. Physiol Entomol 35:46-51

Rund SS, Hou TY, Ward SM, Collins FH, Duffield GE et al (2011) Genome-wide profiling of diel and circadian gene expression in the malaria vector Anopheles gambiae. Proc Natl Acad Sci 108:E421-430

Rund SS, Bonar NA, Champion MM, Ghazi JP, Houk CM, Leming MT, Syed Z, Duffield GE et al (2013) Daily rhythms in antennal protein and olfactory sensitivity in the malaria mosquito Anopheles gambiae. Sci Rep 3:2494

Rund SS, Gentile JE, Duffield GE et al (2013) Extensive circadian and light regulation of the transcriptome in the malaria mosquito Anopheles gambiae. BMC Genomics 14(1):218

Rund SS, O'Donnell AJ, Gentile JE, Reece SE et al (2016) Daily rhythms in mosquitoes and their consequences for malaria transmission. Insects 7:14

Salazar MI, Richardson JH, Sánchez-Vargas I, Olson KE, Beaty BJ et al (2007) Dengue virus type 2: replication and tropisms in orally infected Aedes aegypti mosquitoes. BMC microbiol 7:9

Scaraffia PY, Wells MA (2003) Proline can be utilized as an energy substrate during flight of Aedes aegypti females. J Insect Physiol 49:591-601

Scott TW, Takken W (2012) Feeding strategies of anthropophilic mosquitoes result in increased risk of pathogen transmission. Trends Parasitol 28:114-121

Shapiro LL, Murdock CC, Jacobs GR, Thomas RJ, Thomas MB et al (2016) Larval food quantity affects the capacity of adult mosquitoes to transmit human malaria. P Roy Soc B-Biol Sci 283:20160298

Siju KP, Hill SR, Hansson BS, Ignell R et al (2010) Influence of blood meal on the responsiveness of olfactory receptor neurons in antennal sensilla trichodea of the yellow fever mosquito, Aedes aegypti. J Insect Physiol 56:659-665

Spencer CY, Pendergast TH IV, Harrington LC et al (2005) Fructose variation in the dengue vector, Aedes aegypti, during high and low transmission seasons in the Mae Sot region of Thailand. J Am Mosq Contr 21:177-181

Stanczyk NM, Brugman VA, Austin V, Teran FS, Gezan SA, Emery M, Visser TM, Dessens JT, Stevens W, Smallegange RC, Takken W et al (2019) Species-specific alterations in Anopheles mosquito olfactory responses caused by Plasmodium infection. Sci Rep 9:3396

Syed Z (2015) Chemical ecology and olfaction in arthropod vectors of diseases. Curr Opin Insect Sci 10:83-89

Takken W, Knols BG (1999) Odor-mediated behavior of Afrotropical malaria mosquitoes. Ann Rev Entomol 44:131-157

Takken W, Klowden MJ, Chambers GM et al (1998) Effect of body size on host seeking and blood meal utilization in Anopheles gambiae sensu stricto (Diptera: Culicidae): the disadvantage of being small. J Med Entomol 35:639-645

Takken W, van Loon JJ, Adam W et al (2001) Inhibition of hostseeking response and olfactory responsiveness in Anopheles gambiae following blood feeding. J Insect Physiol 47:303-310 
Takken W, Verhulst NO (2013) Host preferences of blood-feeding mosquitoes. Ann Rev Entomol 58:433-453

Takken W, Stuke K, Klowden MJ et al (2002) Biological differences in reproductive strategy between the mosquito sibling species Anopheles gambiae sensu stricto and An. quadriannulatus. Entomol Exp Appl 103:83-89

Tallon AK, Hill SR, Ignell R et al (2019) Sex and age modulate antennal chemosensory-related genes linked to the onset of host seeking in the yellow-fever mosquito. Aedes aegypti Sci Rep 9:43

Tallon AK, Lorenzo MG, Moreira LA, Martinez-Villegas LE, Hill SR, Ignell R et al (2020) Dengue infection modulates host seeking in Aedes aegypti. PloS Neglect Trop D 14:e0008531

Taparia T, Ignell R, Hill SR et al (2017) Blood meal induced regulation of the chemosensory gene repertoire in the southern house mosquito. BMC Genomics 18:393

Tinbergen N (1952) Derived activities; their causation, biological significance, origin, and emancipation during evolution. Q Rev Biol 27:1-32

Van Handel E (1965) The obese mosquito. J Physiol 181(3):478

Vargo AM, Foster WA (1982) Responsiveness of female Aedes aegypti (Diptera: Culicidae) to flower extracts. J Med Entomol 19:710-718

Vargo AM, Foster WA (1984) Gonotrophic state and parity of nectarfeeding mosquitoes. Mosq News 44:6-10

Vogels CBF, Fros JJ, Pijlman GP, van Loon JJA, Gort G, Koenraadt CJM et al (2017) Virus interferes with host-seeking behaviour of mosquito. J Exp Biol 220:3598-3603

Vrzal EM, Allan SA, Hahn DA et al (2010) Amino acids in nectar enhance longevity of female Culex quinquefasciatus mosquitoes. J insect Physiol 56:1659-1664

Wang G, Carey AF, Carlson JR, Zwiebel LJ et al (2010) Molecular basis of odor coding in the malaria vector mosquito Anopheles gambiae. Proc Nat Acad Sci 107:4418-4423

Wekesa JW, Copeland RS, Mwangi W et al (1992) Effect of Plasmodium falciparum on blood feeding behavior of naturally infected
Anopheles mosquitoes in western Kenya. Am J Trop Med Hyg 47:484-488

Wondwosen B, Birgersson G, Seyoum E, Tekie H, Torto B, Fillinger U, Hill SR, Ignell R et al (2016) Rice volatiles lure gravid malaria mosquitoes. Anopheles arabiensis Sci Rep 6:37930

Wondwosen B, Birgersson G, Tekie H, Torto B, Ignell R, Hill SR et al (2018) Sweet attraction: Sugarcane pollen-associated volatiles attract gravid Anopheles arabiensis. Malar J 17:90

Wondwosen B, Hill SR, Birgersson G, Seyoum E, Tekie H, Ignell R et al (2017) A (maize) ing attraction: Gravid Anopheles arabiensis are attracted and oviposit in response to maize pollen odours. Malar J 16:39

Wynne NE, Lorenzo MG, Vinauger C et al (2020) Mechanism and plasticity of vectors' host-seeking behavior. Curr Opin Insect Sci 40:1-5

Yan J, Martínez-de la Puente J, Gangoso L, Gutiérrez-López R, Soriguer R, Figuerola J et al (2018) Avian malaria infection intensity influences mosquito feeding patterns. Int J Parasitol 48:257-264

Yu BT, Ding YM, Mo XC, Liu N, Li HJ, Mo JC et al (2016) Survivorship and fecundity of Culex pipiens pallens feeding on flowering plants and seed pods with differential preferences. Acta Trop 155:51-57

Zappia SP, Hoi AG, Roitberg BD et al (2015) Energy-state dependent response of Anopheles gambiae to DEET-protected, simulated blood-hosts. J Insect Behav 28:67-76

Publisher's Note Springer Nature remains neutral with regard to jurisdictional claims in published maps and institutional affiliations. 